\title{
Cooperación Universidad - Empresa y su efecto sobre el Desempeño Innovador Empresarial
}

\author{
Cecilia I. Velez ${ }^{1}$, Sergio M. Afcha1, Miguel A. Bustamante ${ }^{2^{\star}}$ \\ (1) CENTRUM Católica Graduate Business School, Calle Daniel Alomia Robles 125-129, Los Álamos \\ Pontificia Universidad Católica del Perú Av. Universitaria 1801, Lima 32, Perú. (e-mail: cvelez@pucp.pe; \\ safcha@pucp.pe) \\ (2) Facultad de Economía y Negocios, Universidad de Talca. Dos Norte 685, 3465548 Talca, Chile. \\ (e-mail: mabu@utalca.cl)
}

* Autor a quien debe ser dirigida la correspondencia.

Recibido Abr. 13, 2018; Aceptado Jun. 12, 2018; Versión final Jul. 18, 2018, Publicado Feb. 2019

\begin{abstract}
Resumen
El objetivo de esta investigación es determinar la relación entre cooperación universidad - empresa y su efecto sobre el desempeño innovador empresarial. El trabajo examina el efecto de la cooperación entre universidad - empresa acerca del desempeño innovador empresarial y determina si el tamaño de la empresa y los gastos en investigación y desarrollo son factores que influyen para desarrollar la cooperación universidad-empresa para la innovación. Se utilizó la encuesta nacional de actividades de innovación del Ecuador año 2013 con una muestra estratificada de 2.815 empresas de 24 provincias del Ecuador. Para el análisis econométrico se utilizó los modelos logit, probit y tobit. Los hallazgos de la investigación indican que la cooperación universidad - empresa influye positivamente en el desarrollo de la innovación empresarial, y existe influencia del tamaño de las empresas en la vinculación universidad- empresa. Sin embargo, esta doble hélice no presenta relación con la inversión en investigación y desarrollo de las empresas en Ecuador.
\end{abstract}

\section{Cooperation between University-Enterprises and its effect on Innovative Business Performance}

\begin{abstract}
The objective of this research is to determine the relationship between university - business cooperation and its effect on entrepreneurial innovation performance. It has three purposes, to examine the effect of universitycompany cooperation on innovative business performance and to determine whether the size of the company and the expenditures on research and development are factors that influence to develop the cooperation university-company for innovation. The national survey of innovation activities of Ecuador was used in 2013 with a stratified sample of 2.815 companies from 24 provinces of Ecuador. The logit, probit and tobit models were used for the econometric analysis. The research findings indicate that university - business cooperation positively influences the development of business innovation, and there is an influence of the size of the companies in the university-business relationship. However, this double helix has no relation with the investment in research. and development of companies in Ecuador.
\end{abstract}

Keywords: cooperation university-business; business development; tobit; logit; probit 


\section{INTRODUCCIÓN}

La cooperación universidad - empresa mejora el desempeño innovador debido a que el conocimiento generado en la universidad contribuye al proceso de innovación de las empresas (Álvarez, 2013; Arias, et al., 2012), cooperación que, en países desarrollados (Aristei et al 2016; Bruneela et al., 2010; Chang et al., 2010) demuestra que la universidad, como fuente de información, tiene un efecto positivo en el desempeño innovador incrementando las cifras de negocio (Molina, et al, 2011). En complemento a lo mencionado, García (2013) encontró que las empresas que usan a la Universidad como fuente de información obtienen un alto desempeño innovador, complementariamente Laursen y Salter (2004) en estudios realizados en el Reino Unido especialmente en industrias químicas, eléctricas y electrónicas de alta intensidad competitiva e intensidad de investigación, encontraron que la intensidad de investigación de las empresas en estas industrias hace que busquen todas las fuentes externas de información y colaboración incluyendo las universidades para desarrollar innovaciones que mejoren su posición competitiva. La interacción universidad - empresa también se produce mediante el acceso a personal calificado (Ponce y Güemes, 2016, Vega, et al., 2011), lo que permite a las empresas compartir riesgos y beneficios (Solleiro y Gaona, 2012) así como acceder progresivamente a los incrementos de complejidad que surge del sector industrial en el que las empresas se desempeñan (Vega-Jurado, et al., 2008).

La teoría de la cooperación universidad - empresa (Álvarez, 2013; Arias, et al., 2012) sostiene que los conocimientos que provienen de la universidad son transferidos a las empresas y aportan a distintos tipos de innovación y competitividad y que permite a las empresas avanzar conjuntamente ante el desafío al que se enfrentan y además, alcanzar niveles superiores de conocimiento (Chang, 2010; Rõigas et al., 2014). Sin embargo, se observan dificultades en la interacción universidad - empresa que surgen de las inciertas y no codificable naturaleza de know-how científico, lo que se traduce en altos costos de transacción y fallos sistémicos en el mercado de este know-how (Veugelers y Cassiman, 2005). La importancia de la cooperación universidad empresa ha motivado la investigación sobre las variables que ayudan a explicar mejor esta relación de cooperación (Ankrah y Al-Tabbaa, 2015; Aristei et al., 2016) a cuyo respecto, Veugelers y Cassiman (2005) propusieron un modelo que identifica diversas variables de control como son el tamaño de la empresa, gastos I+D, si la empresa es local o pertenece a un grupo extranjero y la antigüedad de la empresa; llegándose a concluir que las empresas grandes y más antiguas son las más propensas a colaborar y que la cooperación con las universidades es complementaria a otras actividades de innovación como desarrollar su propio I+D, acceder a información externa y cooperar con otros actores del mercado (Laursen y Salter, 2004; Veugelers y Cassiman, 2005).

En países desarrollados, los factores determinantes de la cooperación universidad - empresa (Bellucci y Pennacchio, 2016; García, 2013; Laursen y Salter, 2004; Veugelers y Cassiman, 2005) identifican el tamaño de la empresa, la capacidad de absorción, la intensidad de gastos de I+D y la antigüedad de la empresa como factores que motivan la cooperación con las universidades en actividades de innovación. En consecuencia, la propensión a formar un acuerdo con un socio académico depende del tamaño absoluto del socio empresarial (Laursen y Salter, 2004), de hecho, las empresas que subcontratan la investigación y el desarrollo y la patente para proteger la innovación, muestran niveles mayores de colaboración. Es en este contexto de relaciones universidad - empresa que surge el modelo triple hélice (Álvarez, 2013; Arias, et al., 2012) como un proceso intelectual encaminado a fomentar estas relaciones y, por otro lado, acreditar la intervención de la universidad en los procesos económicos y sociales, dando pie a una teoría que ha evolucionado y que ubica al Estado como un actor principal en esta vinculación (Chang, 2010).

En países emergentes no existe suficiente evidencia empírica para demostrar que la cooperación universidad - empresa mejora el desempeño innovador (García et al., 2014). Así como tampoco existe suficiente evidencia empírica para demostrar que los factores determinantes que se han identificado en las empresas locales sean factores clave en la cooperación universidad empresa en los países emergentes (García, 2013). Al respecto Zanello et al., (2016) mencionaron que en los países en desarrollo existen barreras para la creación y difusión de las innovaciones como un sistema político débil, características económicas, factores institucionales y factores internos de la empresa, llevando a las empresas a usar menos fuentes externas de información, a ser menos propensas a desarrollar I+D y más propensas a comprar tecnologías.

Como consecuencia de lo mencionado, en los últimos años la comunidad científica ha enfocado su interés en la vinculación Universidad - Empresa, dada su pertinencia y vigencia para el desarrollo de las ciencias empresariales (Bruneel et al., 2010; Chang, 2010; García J., 2013). Sin embargo, la literatura muestra un vacío sobre si la cooperación universidad - empresa mejora el desempeño innovador y efectivamente induce a las empresas a desarrollar actividades de innovación. Por una parte, porque según Zelaya (2010), la universidad actual está en la obligación de convertirse en pilar activo de los nuevos proyectos de innovación que se dan en el entorno empresarial para buscar soluciones, diseñar modelos y estrategias aplicables a la realidad. Por otra parte, porque las empresas que utilizan estrategias abiertas de búsqueda de información, 
dependen del tamaño y antigüedad como factores determinantes de esta cooperación (Álvarez, 2013). En consecuencia, sobre la base conceptual descrita, el presente trabajo busca determinar los factores de cooperación universidad - empresa y su efecto sobre el desempeño innovador empresarial en Ecuador.

\section{METODOLOGÍA}

La presente investigación tiene un enfoque cuantitativo (Gujarati, 2010; Wooldridge, 2010), examina la relación entre la cooperación universidad - empresa y el desempeño innovador empresarial considerando un alcance de carácter explicativo y de corte transversal mediante el uso de los datos de la Encuesta Nacional de Actividades de Innovación 2013, realizada por el Instituto Nacional de Estadísticas y Censos y por la Secretaria Nacional de Educación Superior, Ciencia, Tecnología e Innovación (INEC, 2013).

\section{Hipótesis}

Considerando que la cooperación universidad - empresa afirma que los conocimientos que provienen de la universidad son transferidos a las empresas y aportan a la generación de riqueza (Álvarez, 2013; Arias, et al., 2012), porque permite disminuir riesgos y costos y, por otra, le permite internalizar nuevos conocimientos para mejorar el desempeño innovador (Aristei et al., 2016), la primera hipótesis afirma: H1: La cooperación universidades - empresas influye positivamente en el desempeño innovador de la empresa.

Por otra parte, dado que es más probable que las empresas grandes estén más propensas a utilizar fuentes de conocimientos externos provenientes de las universidades y, que las empresas grandes tienen más recursos para establecer cooperaciones con las Universidades (Bellucci y Pennacchio, 2016; De Moraes et al., 2018; Lin, et al., 2015; López et al., 2015;; Okamuro et al., 2011), la segunda hipótesis señala: H2: Mientras mayor sea el tamaño de la empresa mayor es la posibilidad de establecer cooperación con las universidades para el desarrollo de actividades de innovación. Por último, dado que la cooperación universidad empresa se realiza con la finalidad de internalizar nuevos conocimientos, reducir costos y desarrollar la innovación (Aristei et al., 2016) mediante la gestión de factores clave y determinantes, la tercera hipótesis plantea: H3: Las empresas con altos gastos de I+D tendrán una mayor posibilidad de establecer acuerdos de cooperación con las universidades para el desarrollo de actividades de innovación.

\section{Modelo de análisis y población en estudio}

El estudio se encuentra sustentado en dos modelos cuantitativos (Gujarati, 2010). El primero examina cómo la cooperación universidad empresa influye en el desempeño innovador y, el segundo, examina si el tamaño de la empresa y la intensidad de gastos de I+D, son factores que influyen en las empresas para desarrollar la cooperación universidad - empresas (García, 2013; Laursen y Salter, 2004; Veugelers y Cassiman, 2005).

Tabla 1: Datos descriptivos de la muestra

\begin{tabular}{lr}
\hline \multicolumn{1}{c}{ Tipo de empresa } & Número empresas \\
\hline Explotación de minas y canteras & 74 \\
Industrias manufactureras & 1,191 \\
Suministro de electricidad, gas, vapor y aire acondicionado & 32 \\
Distribución de agua; alcantarillado, gestión de desechos y actividades de saneamiento & 36 \\
Construcción & 171 \\
Comercio al por mayor y al por menor; reparación de vehículos automotores y & 191 \\
motocicletas & 181 \\
Transporte y almacenamiento & 85 \\
Actividades de alojamiento y de servicio de comidas & 209 \\
Información y comunicación & 170 \\
Actividades financieras y de seguros & 49 \\
Actividades inmobiliarias & 278 \\
Actividades profesionales, científicas y técnicas & 70 \\
Actividades de servicios administrativos y de apoyo & 78 \\
Actividades de atención de la salud humana y de asistencia social & 2,815 \\
\hline Total & \\
\hline
\end{tabular}

La base de datos del presente estudio fue extraída de la encuesta de actividades de innovación del Ecuador 2013, la cual fue presentada y aprobada para la investigación por el Programa Doctoral dependiente de CENTRUM de la Pontificia Universidad Católica de Lima, Perú (Julio 2016). 
La población objeto de estudio es de 467.302 empresas de los sectores de manufacturas, minas y canteras, construcción, comercio y servicios de Ecuador, la que fue debidamente verificada en términos de su consistencia por el INEC (2013) mediante una sintaxis para el control del flujo de la encuesta y una malla de validación que garantiza la calidad de los datos. En suma, verificadas las fuentes de datos para el presente trabajo, la muestra inicial fue de 3.188 empresas y luego de la validación realizada por el propio INEC mediante los métodos indicados en el párrafo anterior, la muestra efectiva quedo en 2.815 empresas públicas y privadas (Tabla 1), estratificadas por sector y 24 provincias del Ecuador (INEC, 2013) como se describe en la Tabla 1.

\section{Definición de variables}

Se definió como variables dependientes la cooperación universidad - empresa expresada en la pregunta: ¿Qué importancia tuvieron para las actividades de innovación de la empresa la fuente de información y la cooperación de universidades? El primer modelo consideró con valor cero (0) aquellas empresas con baja o sin utilización de la universidad como fuente de información y de cooperación y el valor de uno (1) las empresas con media y alta importancia de la universidad como fuente de información y de cooperación. En el modelo 1, la variable Cooperación Universidad - Empresa y su efecto en el Desempeño Innovador en las Empresas, se expresa en la fórmula (1).

$$
Y i^{*}=\beta 0+\beta 1 \mathrm{X} 1+\beta 2 \mathrm{X} 2+\beta 3 \mathrm{X} 3+\beta 4 \mathrm{X} 4+\beta 5 \mathrm{X} 5+\mathrm{Ui}
$$

Dónde:

$\mathrm{Yi}^{*}=$ Variable dependiente desempeño innovador empresa

$\beta 0=$ constante

$\mathrm{x} 1=$ Variable independiente colaboración Universidad Empresa

x2 = Tamaño de la empresa

x3 = Gastos I+D

$\mathrm{x} 4=$ Empresa local o pertenece a grupo extranjero

$x 5=$ Antigüedad de la empresa

$\beta 1, \beta 2, \beta 3, \beta 4, \beta 5=$ parámetros

Como segundo modelo se consideró para la variable dependiente los valores ordinales 1 (alta), 2 (media), 3 (baja) y 4 (no utilizado). Complementariamente se tuvo en consideración diversas variables de control como tamaño de la empresa, gastos I+D, empresa local o pertenece a un grupo extranjero, antigüedad de la empresa y número de empleados (García, 2013; Laursen y Salter, 2004; Veugelers y Cassiman, 2005). El modelo 2, las variables que influyen sobre la Cooperación Universidad-Empresa se muestra en la fórmula (2).

$$
Y i^{*}=\beta 0+\beta 1 \mathrm{X} 1+\beta 2 \mathrm{X} 2+\beta 3 \mathrm{X} 3+\beta 4 \mathrm{X} 4+\mathrm{Ui}
$$

\section{Dónde:}

$\mathrm{Yi}^{*}=$ Variable dependiente Cooperación Universidad empresa

$\beta 0=$ constante

$\mathrm{x} 1$ = Tamaño de la empresa

$\mathrm{x} 2=$ Gastos $\mathrm{I}+\mathrm{D}$

$\mathrm{x} 3=$ Empresa local o pertenece a grupo extranjero

$\mathrm{x} 4=$ Antigüedad de la empresa

$\beta 1, \beta 2, \beta 3, \beta 4=$ parámetros

\section{Procedimiento para la validación de hipótesis}

Para la verificación de H1: "La cooperación universidades - empresas influye positivamente en el desempeño innovador de la empresa", se determinó lo siguiente: La variable dependiente, desempeño innovador se mide mediante dos categorías. La medida 1, corresponde al porcentaje de las ventas de nuevos productos 0 productos mejorados en el mercado. La medida 2, toma el valor de 1 si la empresa introdujo al mercado un nuevo o significativamente mejorado producto y 0 en caso contrario. En consecuencia, para esta verificación de hipótesis, se realizan dos ejecuciones una por cada medida. Si existe un efecto positivo y estadísticamente significativo se considerara que existe evidencia empírica para aceptar la hipótesis 1.

Para la primera ejecución que considera la medida 1 se analiza que la variable dependiente, porcentaje de las ventas de nuevos productos o productos mejorados en el mercado, es un valor positivo mayor a cero (0). Se utiliza el modelo Tobit de elección discreta y la variable dependiente es un valor positivo mayor a cero y 
por lo tanto la variable dependiente es censurada (García, 2013; Laursen y Salter, 2004; Veugelers y Cassiman, 2005). Para la segunda ejecución que considera la medida 2, presenta una variable dependiente binaria que puede tomar el valor cero (0) o uno (1) y que los índices de regresión no son aleatorios, se utilizó el modelo Probit de elección discreta para variables dependientes binarias (García, 2013; Laursen y Salter, 2004; Veugelers y Cassiman, 2005).

Las variables independientes tomarán valor uno (1) cuando en la encuesta se marque a nivel alta o media la innovación que tuvo como fuente de información y de cooperación la Universidad y tomará cero (0) cuando en la encuesta se marque con baja o no utilizada la innovación que tuvo como fuente de información la cooperación con la Universidad. Se utilizaron como variables de control, el tamaño de la empresa y los gastos I+D (Laursen y Salter, 2004; Veugelers y Cassiman, 2005). Para el tamaño de la empresa se utiliza como medida el número de empleados de la empresa y para la variable Gastos I+D utiliza como medida el nivel de los gastos dividido para las ventas en el periodo analizado.

Complementariamente, las variables empresa local o pertenece a un grupo extranjero, se define como variable Dummy que toma valor de uno (1) si la empresa es local y cero (0) si pertenece a grupo extranjero. La variable Antigüedad de la empresa toma el valor de uno (1) si la empresa es antigua (mayor a cinco años) y cero (0) si es menor de 5 años el inicio de sus actividades en el país. En la primera medida se utilizara el modelo Tobit, definido por la fórmula (3).

$$
Y i^{*}=\beta 0+\beta 1 \mathrm{X} 1+\beta 2 \mathrm{X} 2+\beta 3 \mathrm{X} 3+\beta 4 \mathrm{X} 4+\beta 5 \mathrm{X} 5+\mathrm{Ui}
$$

Dónde:

$\mathrm{Yi}^{*}=$ Variable dependiente el $\%$ de las ventas de nuevos productos o productos mejorados en el mercado

$\beta 0=$ constante

$\mathrm{x} 1$ = Variable independiente colaboración Universidad Empresa

x2 = Tamaño de la empresa

x3 = Gastos I+D

$\mathrm{x} 4$ = Empresa local o pertenece a grupo extranjero

$x 5$ = Antigüedad de la empresa

$\beta 1, \beta 2, \beta 3, \beta 4, \beta 5=$ parámetros

La función de probabilidad del modelo Tobit se muestra en la fórmula (4).

$$
\log L=\sum_{Y i>0}-\frac{1}{2}\left[\log (2 \pi)+\log \sigma^{2}+\frac{(y i-\beta x i)^{2}}{\sigma^{2}}\right]+\sum_{y i=0} \log \left[1-F\left(\frac{\beta x i}{\sigma}\right)\right]
$$

En la segunda medida se ejecuta el modelo Probit que se muestra en la fórmula (5).

$$
Y i^{*}=\beta 0+\beta 1 \mathrm{X} 1+\beta 2 \mathrm{X} 2+\beta 3 \mathrm{X} 3+\beta 4 \mathrm{X} 4+\beta 5 \mathrm{X} 5+\mathrm{Ui}
$$

Dónde:

$\mathrm{Yi}^{*}=$ Variable dependiente desempeño innovador empresa

$\beta 0=$ constante

$\mathrm{x} 1$ = Variable independiente colaboración Universidad Empresa

x2 = Tamaño de la empresa

x3 = Gastos I+D

x4 = Empresa local o pertenece a grupo extranjero

$x 5$ = Antigüedad de la empresa

$\beta 1, \beta 2, \beta 3, \beta 4, \beta 5=$ parámetros

Para el modelo Probit se utiliza el procedimiento de máxima verosimilitud (MV), considerando una muestra de $\mathrm{n}$ observaciones independientes. La estimación MV se maximiza utilizando la probabilidad de la función de registros asociados en la fórmula (6).

$$
Q(\beta)=\sum_{i=1}^{N}\left[y i \ln F\left(x_{i}^{1} \beta\right)+(1-y i) \ln \left\{1-F\left(x_{i}^{1} \beta\right)\right\}\right]
$$

Dado que la estimación de máxima verosimilitud se obtiene por métodos iterativos normalmente distribuidos, 
se procedió a obtener los coeficientes de regresión de las variables y su respectivo estadístico Wald con su asociado $p$ value, verificando que los coeficientes de la regresión tengan significancia estadística menor al 0.05. Una vez realizado los cálculos y verificada las hipótesis se procedió al análisis de los resultados proporcionados por los modelos Probit y Tobit. Sobre la base de las descripciones metodológicas indicadas, se procedió a la verificación, prueba o rechazo de las hipótesis: H2: "Mientras mayor sea el tamaño de la empresa mayor es la posibilidad de establecer cooperación con las universidades para el desarrollo de actividades de innovación" y H3: "Las empresas con altos gastos de I+D tendrán una mayor posibilidad de establecer acuerdos de cooperación con las universidades para el desarrollo de actividades de innovación". Para estas verificaciones, se resolvió utilizar el modelo Logit que se define en la fórmula (7).

$$
Y i^{*}=\beta 0+\beta 1 \mathrm{X} 1+\beta 2 \mathrm{X} 2+\beta 3 \mathrm{X} 3+\beta 4 \mathrm{X} 4+\mathrm{Ui}
$$

Dónde:

$\mathrm{Yi}^{*}=$ Variable dependiente Cooperación Universidad empresa

$\beta 0=$ constante

$\mathrm{x} 1$ = Tamaño de la empresa

$\mathrm{x} 2=$ Gastos $\mathrm{I}+\mathrm{D}$

x3 = Empresa local o pertenece a grupo extranjero

$\mathrm{x} 4$ = Antigüedad de la empresa

$\beta 1, \beta 2, \beta 3, \beta 4, \beta 5=$ parámetros

La estimación por el método de máxima verosimilitud se muestra en la fórmula (8).

$$
\log L=\sum y i \ln \left[\frac{e^{\alpha+\beta x}}{1+e^{\alpha+\beta X}}\right]+\sum(1-y i) \ln \left[1-\frac{e^{\alpha+\beta x}}{1+e^{\alpha+\beta X}}\right]
$$

Por último, la Tabla 2 demuestra la composición de las variables utilizadas en el estudio y, como se observa, existen rangos y bordes de las fuentes para la innovación que definen la relación universidad - empresa como fuente de información y conocimiento para la innovación.

\begin{tabular}{|c|c|c|c|}
\hline Nombre & Nomenclatura & Composición & Tipo de Variable \\
\hline $\begin{array}{l}\text { La Empresa ha } \\
\text { Innovado }\end{array}$ & III_1_a & $1=$ Si ha Innovado $0=$ No ha Innovado & Binomial \\
\hline Porcentaje de Ventas & Y2_porc & $\begin{array}{c}\text { Promedio de Ventas }_{\mathrm{i}} \\
\text { Promedio Total de Ventas } \\
\mathrm{i}=\text { empresa }(1,2,3, \ldots, \mathrm{n})\end{array}$ & Continua \\
\hline $\begin{array}{c}\text { Cooperación } \\
\text { Universidad Empresa }\end{array}$ & VIII_2_e & $\begin{array}{c}1=\text { Si Existe Cooperación } \\
0=\text { No Existe Cooperación }\end{array}$ & Binomial \\
\hline Tamaño de la Empresa & Tamaño & $\begin{array}{c}1=\text { Microempresa } 2=\text { Pequeña } \\
3=\text { Mediana } 4=\text { Grande }\end{array}$ & Multinomial \\
\hline I\&D & RatioID_VTA & $\frac{\text { Promedio gasto I\&D }}{\text { Promedio ventas }}$ & Continua \\
\hline Empresa Local & Local & $\begin{array}{c}1=\text { Empresa Local } \\
0=\text { Empresa Extranjera }\end{array}$ & Binomial \\
\hline Antigüedad & Antiguedad_d & $\begin{array}{c}1=\text { Antigua (> } 5 \text { años }) \\
0=\text { Nueva }(<5 \text { años })\end{array}$ & Binomial \\
\hline
\end{tabular}

Tabla 2: Composición de Variables

\section{RESULTADOS}

Respecto de $\mathrm{H} 1$, que afirma que la cooperación universidades - empresas influye positivamente en el desempeño innovador de la empresa, se aplicaron los Modelos Probit y Tobit, cuyos parámetros se muestran en la Tabla 3. Del primer modelo, Tobit (1), se aprecia que tres de las cinco variables explicativas planteadas en el modelo son significativas al 1\%, las cuales son la Cooperación Universidad Empresa, tamaño de la empresa, y gasto de investigación y desarrollo. Las variables que no fueron significativas a ningún nivel de significancia fueron, existencia de empresas locales en el modelo y antigüedad de las empresas. Utilizando la formula (3), los parámetros obtenidos mediante Tobit (1) se entregan en la expresión (9). Por otra parte, los parámetros obtenidos del modelo Probit (1) se muestran la expresión (10): 
Desemp Innv $=-0.090+0.350$ Cooperación Univ Emp +0.038 Tamaño empresa +0.738 Ratio

ID/Venta - 0.098 Empresa local o ext - 0.112 Antigüedad empresa + Ui

Desemp Innv $=-1.828+0.255$ Cooperación Univ Emp + 0.216 Tamaño empresa + 1.392 Ratio

ID/Venta + 0.086 Empresa local o ext - 0.189 Antigüedad empresa + Ui

Tabla 3: Modelos Econométricos

\begin{tabular}{|c|c|c|c|c|}
\hline Variables & Tobit (1) & Tobit (2) & Probit (1) & Probit (2) \\
\hline Cooperación Univ-Emp & $\begin{array}{c}0.350^{* * *} \\
(7.73)\end{array}$ & $\begin{array}{c}0.350^{* * *} \\
(7.72)\end{array}$ & $\begin{array}{c}0.255^{*} \\
(1.73)\end{array}$ & $\begin{array}{l}0.257^{\star} \\
(-1.74)\end{array}$ \\
\hline Tamaño empresa & $\begin{array}{c}0.038^{\star * *} \\
(-3.51)\end{array}$ & $\begin{array}{c}0.035^{\star \star *} \\
(-3.31)\end{array}$ & $\begin{array}{c}0.216^{* * *} \\
(5.84)\end{array}$ & $\begin{array}{c}0.206^{* * *} \\
(5.75)\end{array}$ \\
\hline Ratio ID/Venta & $\begin{array}{c}0.738^{\star * *} \\
(3.98)\end{array}$ & $\begin{array}{c}0.767^{\star \star \star} \\
(4.14)\end{array}$ & $\begin{array}{c}1.392^{* *} \\
(3.05)\end{array}$ & $\begin{array}{c}1.428^{\star *} \\
(3.13)\end{array}$ \\
\hline Empresa local & $\begin{array}{l}-0.098 \\
(-0.19)\end{array}$ & ------ & $\begin{array}{l}0.086 \\
(0.53)\end{array}$ & ------ \\
\hline Antigüedad empresa & $\begin{array}{l}-0.112 \\
(-2.42)\end{array}$ & ------ & $\begin{array}{l}-0.189 \\
(-1.22)\end{array}$ & ------ \\
\hline Constante & $\begin{array}{l}-0.090 \\
(-1.20)\end{array}$ & $\begin{array}{c}-0.200^{* * *} \\
(-6.65)\end{array}$ & $\begin{array}{c}-1.828^{\star * *} \\
(-7.33)\end{array}$ & $\begin{array}{c}-1.899^{\star * *} \\
(-18.25)\end{array}$ \\
\hline Número de Observaciones & 2815 & 2815 & 2815 & 2815 \\
\hline LR Chi2 & 102.00 & 96.18 & ------ & ------ \\
\hline Wald Chi2 & ------ & ------ & 48.36 & 46.25 \\
\hline Pseudo R2 & 0.02 & 0.02 & 0.02 & 0.02 \\
\hline
\end{tabular}

En la estimación del segundo modelo Tobit (2), se excluyen las variables que no son significativas para determinar si las variables que se presentaron significativas en el primer modelo continúan con la misma conducta. Para verificar el cumplimiento de la hipótesis (H1) se observa que en el modelo Tobit (2), el coeficiente de la variable Cooperación Universidad Empresa posee una relación positiva y directa con la variable dependiente de innovación empresarial, lo que indica que entre mayor sea el grado de cooperación entre las Universidades y las Empresas se generará un mejor desempeño innovador en las empresas.

Para la aceptación o rechazo de la hipótesis se observaron las estimaciones del modelo Probit (1), donde se indica que las variables significativa son el tamaño de las empresas y el gasto en investigación y desarrollo. Sin embargo, la variable de cooperación Universidad Empresa, es significativa al 10\% lo que implica que la variable no debe ser rechazada en su totalidad. Por ello, se estimó el modelo Probit (2) donde se aprecia que la variable de cooperación Universidad Empresa continúa con el comportamiento que obtuvo en el modelo Probit (1). Comparando la conducta de los estimadores de las variables en los dos modelos Tobit y Probit, se prueba $\mathrm{H} 1$, en consecuencia, existe incidencia de la cooperación universidad - empresa sobre el mejoramiento del desempeño de la innovación de las empresas en Ecuador. En torno a H2, que afirma que mientras mayor sea el tamaño de la empresa mayor es la posibilidad de establecer cooperación con las universidades para el desarrollo de actividades de innovación, se presenta la Tabla 3 que proporciona los datos para cumplimentar la ecuación paramétrica del modelo Logit. Los parámetros determinados por la formula (2) mediante el modelo Logit (1) se entregan la fórmula (11).

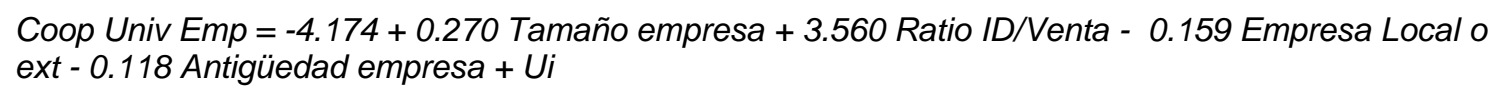

Analizando los resultados del modelo Logit (1) para determinar aprobación o rechazo de H2, se observa que la única variable que resulta significativa es tamaño de las empresas. Además, con la finalidad de aseverar si el estimador mantiene su nivel de significancia, se procedió a aplicar el modelo Logit (2), donde la variable continua sigue siendo significativa. Además, se observa que la variable presenta un coeficiente con signo positivo, es decir que, mientras mayor sea el tamaño de las empresas mayor será la cooperación de las Universidades para el desarrollo de las actividades de innovación. En consecuencia, se aprueba H2.

Para verificar la aprobación o rechazo de H3, que afirma: las empresas con altos gastos de I+D tendrán una mayor posibilidad de establecer acuerdos de cooperación con las universidades, para el desarrollo de actividades de innovación, se analizan los datos de la Tabla 4. Los parámetros determinados por la formula (2) y sobre la base del modelo Logit (1), se muestran en la fórmula (12).

Coop Univ Emp = -4.174 + 0.270 Tamaño empresa + 3.560 Ratio ID/Venta - 0.159 Emp local o ext - 0.118 Antigüedad empresa + Ui 
Tabla 4: Modelos Econométricos

\begin{tabular}{lcc}
\hline Variables & Logit (1) & Logit (2) \\
\hline Tamaño empresa & $0.270^{\star *}$ & $0.246^{\star *}$ \\
& $(2.50)$ & $(2.34)$ \\
Ratio ID/Venta & 3.560 & ----- \\
& $(1.46)$ & \\
Local o externa & 0.159 & ----- \\
& $(0.34)$ & \\
Antigüedad empresa & -0.118 & ---- \\
& $(0.20)$ & $-3.804^{\star \star *}$ \\
Constante & $-4.174^{\star * *}$ & $(-12.52)$ \\
\hline Número de Observaciones & $(-4.91)$ & 2815 \\
LR Chi2 & 2815 & ----- \\
Wald Chi2 & ----- & 5.46 \\
Pseudo R2 & 8.20 & 0.006 \\
\hline
\end{tabular}

Se puede observar que la estimación de parámetros del modelo Logit (1) muestra que la variable independiente Ratio ID/Venta resulta no significativa a ningún nivel de significancia, por lo tanto, las empresas con altos gastos de I+D no tendrían una mayor posibilidad de establecer acuerdos de cooperación con las universidades para el desarrollo de actividades de innovación en Ecuador. En consecuencia, se rechaza H3.

\section{DISCUSIÓN}

En general, en el contexto ecuatoriano, no se aprecia investigación previa sobre la creación de innovación empresarial producto del vínculo universidad - empresa ni siquiera que exista proximidad de relación (HewittDundas, 2011), de manera que la presente investigación es un primer estudio que establece este vínculo mediante la aplicación del modelo Laursen y Salter (2004). Para ello se utiliza el modelo de investigación en innovación de las empresas de Schwartz y Guaipatín (2014) y se determinan las variables que influyen en la innovación empresarial en Ecuador. Sin embargo, no existe especificación de las variables que explican el crecimiento de la innovación empresarial como lo sugieren los estudios de la relación empresa - universidad (Bruneel et al., 2010).

Los resultados colaborar a confirmar la existencia de factores que explican el por qué las empresas utilizan las universidades para acceder a innovación y desarrollo (Cassiman et al., 2010; Gómez et al., 2014). Se observa que la intensidad de $I+D$, el tamaño de la empresa y el medio ambiente industrial validados metodológicamente (Gujarati, 2010) son factores importantes para explicar la propensión de las empresas a utilizar las universidades en sus actividades innovadoras confirmando las apreciaciones de Laursen y Salter (2004). Se ratifican así mismo otros estudios que examinaron el tamaño de la empresa como un factor de propensión para la cooperación (Bellucci y Pennacchio, 2016; De Moraes et al., 2018; Lin, et al., 2015).

El contraste metodológico realizado entre los modelos Tobit y Probit (Wooldridge, 2010), muestra que la variable exógena de cooperación universidad empresa incide significativamente de forma directa en el desempeño innovador empresarial. Lo anterior es consistente con la literatura reciente (Vega-Jurado, et al., 2017) ya que la contribución directa de las universidades con la empresa es muy probable que esté altamente relacionada con un reducido número de sectores industriales, entre las empresas que tienen capacidad y entre las empresas que han adoptado un enfoque de búsqueda de innovación abierta (Castro et al., 2010; Laursen y Salter, 2004). Complementariamente, las técnicas econométricas (Wooldridge, 2010) muestran la incidencia directa que existe entre el tamaño empresarial con la probabilidad de cooperación con las universidad para el desarrollo de actividades de innovación (García, 2013; Laursen y Salter, 2004; Veugelers y Cassiman, 2005). Así mismo, el modelo Logit permite afirmar que el tamaño de las empresas es efectivamente una variable independiente y significativa en la estimación del modelo. Además, el mismo modelo Logit, comprueba que los altos gastos de Investigación y Desarrollo no poseen un valor probabilístico significativo para establecer acuerdos de cooperación con las Universidades para el desarrollo de actividades de innovación, puesto que la variable independiente de gasto en I+D, resultó no significativa.

Los resultados demuestran que las teorías de la cooperación universidad - empresa para la innovación (Morales, et al., 2015) en particular las realizadas en países desarrollados (Ankrah y Al-Tabbaa, 2015) no son totalmente aplicables al Ecuador ya que esta cooperación sí incrementa el desempeño innovador de las empresas y son las grandes empresas las que tienen mayor propensión a desarrollar esta cooperación, sin embargo los gastos en I+D no son un factor que influya en las empresas para desarrollar cooperación 
universidad - empresa. Por último, los gastos de I+D tampoco son un factor determinante para desarrollar la cooperación universidad empresa en países emergentes (Garcia et al., 2014). A fin de ampliar los hallazgos de este estudio, se recomienda realizar futuras investigaciones sobre la cooperación universidad - empresa en países emergentes a fin de establecer comparaciones significativas con empresas de países en desarrollo.

\section{CONCLUSIONES}

En general, los resultados confirman la existencia de relaciones de cooperación universidad - empresa para el desempeño innovador empresarial en Ecuador. Esto se expresa en los índices de tamaño de la empresa y ratio de ventas que alcanzan valores positivos, estableciendo un efecto directo sobre la variable dependiente de cooperación para la innovación.

En lo particular, se comprueba por los modelos Tobit, Probit y Logit, la significancia estadística de la variable, tamaño de las empresas, en la generación de una mayor probabilidad de establecer cooperación con la universidad, sin embargo, esto no se ratifica de igual manera para la variable ratio de venta, la cual no resulta significativa en todos los casos.

Aunque se comprueba la incidencia de la cooperación universidad empresa en el desempeño innovador y por lo tanto se aprueba $\mathrm{H} 2$ que afirma que mientras mayor sea el tamaño de la empresa mayor es la posibilidad de establecer cooperación con las universidades para el desarrollo de actividades de innovación; se rechaza H3 que sugiere que las empresas con altos gastos de I+D tendrán una mayor posibilidad de establecer acuerdos de cooperación con las universidades para el desarrollo de actividades de innovación, por cuanto esto no se ratifica mediante la significancia estadística de la variable gastos de Investigación y Desarrollo l+D, en la generación de una mayor probabilidad de establecer cooperación con la universidad.

\section{REFERENCIAS}

Álvarez, M.E., Vinculación Universidad-Sociedad: Estudio de un Equipo de Trabajo en el Área de Gestión Ambiental de la Universidad Nacional de La Plata. Tesis de Grado. Universidad Nacional de La Plata. Facultad de Humanidades y Ciencias de la Educación En Memoria Académica (2013)

Ankrah, S. y O. Al-Tabbaa, Universities-Industry Collaboration: A Systematic Review, Scandinavian Journal of Managment, 387-408 (2015)

Arias, L., M. Portilla y S. Fernandez, Vinculación Universidad Empresa Como Formador de Capital Humano para la Competitividad e Impacto Social, Scientia et Technica Año XVII, Universidad Tecnológica de Pereira, ISSN: 0122-1701, 52, 57-64 (2012)

Aristei, D., M. Vecchi y F. Venturini, University and Inter-Firm R\&D Collaborations: Propensity And Intensity fo Cooperation In Europe, The Journal of Technology Transfer, 41(4), 841-871 (2016)

Bellucci, A. y L. Pennacchio, University knowledge and firm innovation: evidence from European countries, The journal of technology transfer, 41(4), 730-752 (2016)

Bruneel, J., P. D'Este y A. Salter, Investigating the Factors that Diminish the Barriers to University-Industry Collaboration. Research Policy, 858 (2010)

Bruneela, J., P. D'Esteb y A. Salter, Investigating the Factors that Diminish the Barriers to University-Industry, Research Policy, 858-868 (2010)

Cassiman, B., M. Di Guardo y G. Valentini, La Organización de los Vínculos con la Ciencia: ¿Cooperar o Contrato?: Un Análisis a Nivel del Proyecto, Research Policy, 39, 882-892 (2010)

Castro, E. y J. Sutz, Universidad, Conocimiento e Innovacion. Ciencia, Tecnologia y Universidad en Iberoamerica, 103$119(2010)$

Chang, H., El Modelo de la Triple Hélice como un Medio para la Vinculación entre la Universidad y Empresa, Revista Nacional de Administración, 85-93 (2010)

De Moraes Silva, D.R., A.T. Furtado y N.S. Vonortas, University-industry R\&D cooperation in Brazil: a sectoral approach, The Journal of Technology Transfer, 43(2), 285-315 (2018)

García, F.J. Does Inward Foreign Direct Investment Improve The Innovative Performance of Local Firms? Research Policy, (42) 231-244 (2013)

García, O., J. Quintero y J. Arias, Capacidades de Innovación, Desempeño Innovador y Desempeño Organizacional en Empresas del Sector Servicios, Cuadernos de Administración, 27 (49), 87-108 (2014)

Gomez, F., T. Daim y J. Robledo, Characterization of the Relationship Between Firms and Universities and Innovation Performance: The Case of Colombian Firms, Journal of Technology Management \& Innovation, 9, 70-83 (2014)

Gujarati, D.N. y D.C. Porter, Econometria, Mexico, McGrawHill (2010) 
Hewitt-Dundas, N., El Papel de la Proximidad en la Cooperación Universidad-Empresa para la Innovación, The Journal of Technology Transfer, (38), 93-115 (2011)

INEC, Metodología de la Encuesta Nacional de Actividades de Innovación (Al): 2009-2011, Guayaquil, INEC (2013)

Jaramillo, I.E.P. y D.G. Castorena, Identification of key factors of academia in the process of linking in the triple helix of innovation model in Mexico, a state of the art matrix, Nova scientia, 8(16), 246-277 (2016)

Laursen, K. y A. Salter, Searching High and Low: What Types of Firms Use Universities, Researcho Policy, 1201-1215 (2004)

Lin, T.C., S.F. Kung y H. C. Wang, Effects of firm size and geographical proximity on different models of interaction between university and firm: A case study, Asia Pacific Management Review, 20(2), 90-99 (2015)

López, S.F., B.P. Astray, D.R. Pazos y N. Calvo, Are Firms Interested in Collaborating with Universities? An openInnovation Perspective in Countries of the South West European Space, Service Business, 9 (4), $637-662$ (2015)

Molina, F., M. Martínez y V. Jasmine, The Dark Side of Trust: the Benefits, Costs and Optimal Levels of Trust for Innovation Performance, Journal of Agribusiness in Developing and Emerging Economies, 1 (1), 75-96 (2011)

Morales, M., P. Sanabria y D. Caballero, Caracteristicas de la Vinculacion Universidad-Entorno En La Universidad Nacional De Colombia. Rev. Fac. Cienc. Econ, XXIII (1), 189-208 (2015)

Okamuro, H., M. Kato e Y. Honjo, Determinants of R\&D Cooperation in Japanese Start-Ups. Research Policy, 40 (5), 728 $738(2011)$

Ponce Jaramillo, I. E. y D. Güemes Castorena, Identificación de factores clave de la academia en el proceso de vinculación del modelo de la triple hélice de la innovación en México, Nova Scientia, 8(16), 246-277 (2016)

Rõigas, K., M. Seppo, U. Varblane y P. Mohnen, Which Firms use Universities as Cooperation Partners? - The Comparative View in Europe, Social Science Research Network, 3-28 (2014)

Schwartz, L. y C. Guaipatín, Ecuador: Análisis del Sistema Nacional de Innovación: Hacia la Consolidación de una Cultura Innovadora (2014)

Solleiro, J. L. y C. Gaona, Promotion of a regional innovation system: the case of the State of Mexico, Procedia-Social and Behavioral Sciences, 52, 110-119 (2012)

Vega, J., L. Manjarrés, E. Castro e I. Fernández, Las Relaciones Universidad-Empresa: Tendencias y Desafíos en el Marco del Espacio Iberoamericano del Conocimiento, Revista Iberoamericana de Educación, 109-124 (2011)

Vega-Jurado, J., A. Gutiérrez-Gracia e I. Fernández-de-Lucio. ¿Cómo innovan las empresas españolas?: Una evidencia empírica, Journal of technology management \& innovation, 3(3), 100-111 (2008)

Vega-Jurado, J., S. nKask y L. Manjarrés-Henriquez, University industry links and product innovation: cooperate or contract? Journal of technology management \& innovation, 12(3), 1-8 (2017)

Veugelers, R. y B. Cassiman, R\&D Cooperation Between Firms and Universities, Some Empirical Evidence from Belgian Manufacturing, International Journal of Industrial Organization, 355-379 (2005)

Wooldridge, J.M., Introducción a la Econometría - un Enfoque Moderno, México D.F., Cengage Learning Editores S.A. (2010)

Zanello, G., X. Fu, P. Mohnen y M. Ventresca, The Creation and Diffusion of Innovation in Developing Countries, A Systematic Literature Review, Journal of Economic Surveys, 30 (5), 884-912 (2016)

Zelaya, J. R., La Vinculación Universidad-Empresa y la Provisión de Servicios Tecnologicos, 1ª Ed., Vol. 1, San Salvador, San Salvador, UFG Editores (2010) 